\title{
Article \\ A Deep-Learning-Based Meta-Modeling Workflow for Thermal Load Forecasting in Buildings: Method and a Case Study
}

\author{
Yuhao Zhou ${ }^{1,2,3,+}$, Yumin Liang ${ }^{4, *,+}\left(\mathbb{D}\right.$, Yiqun Pan ${ }^{4, *}$, Xiaolei Yuan ${ }^{4}$, Yurong Xie ${ }^{1,2,3}$ and Wenqi Jia 5 \\ 1 Huadian Electric Power Research Institute Co., Ltd., Hangzhou 310030, China; yuhao-zhou@chder.com (Y.Z.); \\ yurong-xie@chder.com (Y.X.) \\ 2 National Energy Distributed Energy Technology R\&D Center, Hangzhou 310030, China \\ 3 Key Laboratory of Energy Storage and Building Energy-Saving Technology of Zhejiang Province, \\ Hangzhou 310030, China \\ 4 School of Mechanical Engineering, Tongii University, Shanghai 201804, China; damon01_yuan@tongji.edu.cn \\ 5 Department of Mechanical Engineering, Texas A\&M University, College Station, TX 77840, USA; \\ hubertjwq@tamu.edu \\ * Correspondence: yumin_liang@tongji.edu.cn (Y.L.); yiqunpan@tongji.edu.cn (Y.P.) \\ + These authors contributed equally to this work.
}

Citation: Zhou, Y.; Liang, Y.; Pan, Y.; Yuan, X.; Xie, Y.; Jia, W. A Deep-Learning-Based Meta-Modeling Workflow for Thermal Load Forecasting in Buildings: Method and a Case Study. Buildings 2022, 12, 177. https://doi.org/10.3390/ buildings12020177

Received: 13 January 2022

Accepted: 31 January 2022

Published: 4 February 2022

Publisher's Note: MDPI stays neutral with regard to jurisdictional claims in published maps and institutional affiliations.

Copyright: (C) 2022 by the authors. Licensee MDPI, Basel, Switzerland. This article is an open access article distributed under the terms and conditions of the Creative Commons Attribution (CC BY) license (https:// creativecommons.org/licenses/by/ $4.0 /)$.

\begin{abstract}
This paper proposes a meta-modeling workflow to forecast the cooling and heating loads of buildings at individual and district levels in the early design stage. Seven input variables, with large impacts on building loads, are selected for designing meta-models to establish the MySQL database. The load profiles of office, commercial, and hotel models are simulated with EnergyPlus in batches. A sequence-to-sequence (Seq2Seq) model based on the deep-learning method of a one-dimensional convolutional neural network (1D-CNN) is introduced to achieve rapid forecasting of all-year hourly building loads. The method performs well with the load effective hour rate (LEHR) of around $90 \%$ and MAPE less than $10 \%$. Finally, this meta-modeling workflow is applied to a district as a case study in Shanghai, China. The forecasting results well match the actual loads with $\mathrm{R}^{2}$ of 0.9978 and 0.9975 , respectively, for the heating and cooling load. The LEHR value of all-year hourly forecasting loads is $98.4 \%$, as well as an MAPE of $4.4 \%$. This meta-modeling workflow expands the applicability of building-physics-based methods and improves the time resolution of conventional data-driven methods. It shows small forecasting errors and fast computing speed while meeting the required precision and convenience of engineering in the building early design stage.
\end{abstract}

Keywords: building load forecasting; meta-modeling; deep learning; CNN; Seq2Seq; district

\section{Introduction}

Urbanization brings the rapid growth of the building floor area and also inevitably causes an explosive increase in energy consumption [1,2]. The building sector is responsible for over one-third of global final energy consumption and nearly $40 \%$ of total $\mathrm{CO}_{2}$ emissions [3], which has directly put great pressure on the natural environment and resources. Building energy conservation has become one of the keys to achieve sustainable development on an urban scale [4]. In this context, more buildings are designed and constructed in districts with district energy systems, including communities, blocks, and other areas below the city scale. A district energy system can lead to remarkable environmental and economic benefits [5], such as facilitating renewable energy utilization and waste heat recovery by combined heat and power (CHP), replacing the less efficient boilers in individual buildings with a more efficient central heating system, the ability to integrate a large-scale energy storage system, and the economies of district heating and cooling applications.

A district energy system has many advantages with the premise of reasonable design, which needs accurate forecasting of the district building thermal loads to meet the all-time demand [6]. Considering the dynamic coupling of time and space, the district building 
loads are not the simple addition of individual building loads. In general, maximum cooling and heating loads at a district scale will be smaller than the static superposition of single building loads, which easily causes system over-configuration and inefficient operation. As building load forecasting is significant to the district energy system in the early design stage, researchers around the world have put forward many forecasting methods, which can be roughly categorized into two types: the building-physics-based method and data-driven method [7].

The building-physics-based method [8,9] is to establish a building-physics model and complete numerical analysis using building-simulation programs, which requires physically measurable variables to describe buildings and technologies in detail. The input data for the building-physics-based method includes building information, thermal characteristics of envelopes, outdoor and indoor temperatures, ventilation rates, energy consumption of appliances, the number of occupants, etc. [10]. Considering that detailed input information may sometimes be unavailable, simplified prototypical building models are commonly modeled to represent specific buildings in a particular district or even in a city [11]. Heiple and Sailor [11] used eQUEST to establish a set of prototypical building models, and estimated detailed energy consumption profiles of different building types. Existing buildings of most large U.S. cities are matched to building prototypes with a geographical information system (GIS) so that their energy consumption can be calculated by multiplying the corresponding prototype's energy use intensity by the actual building's floor space. A case study in Houston revealed that the prototypical models can accurately capture the actual building energy consumption [11]. However, the time-consuming process of simulating a set of prototype buildings is the largest shortcoming of this method, which limits the feasibility and extensibility of physics-based models.

The data-driven method applies statistical theory to mine the deep relationships between historical load data and building feature variables, so as to establish the load forecasting model. Scholars have studied data-driven methods and put forward many models, including the regression analysis model [12-15], time series analysis model [16,17], support vector machine (SVM) [18,19], artificial neural network (ANN) [20,21], and so on. Among data-driven methods, the artificial neural network (ANN) has an excellent ability to deal with non-linear problems, which has become one of the main methods of building load forecasting. Hou et al. [22] applied rough set (RS) theory to find relevant parameters used as an input of an ANN to forecast an air-conditioning cooling load. As more effective information is used, the synthesized RS-ANN model improves the accuracy and the robustness of load forecasting results [22]. However, the data-driven methods lack a physical description of the real system, and there is no clear connection between the model inputs and the building features. Through review, it is also found that the time resolution is relatively rough in current data-driven methods, in which historical data are mainly used to forecast the peak building load $[13,16]$ or the short-term loads $[13,14,17,19]$. However, the all-year hourly load profile is more meaningful in the building design stage. Only the dynamic superposition of all-year hourly loads in district or urban-scale buildings can accurately determine the peak load value and the overall load profiles.

In view of the above shortcomings of conventional methods, this paper puts forward a meta-modeling workflow to forecast all-year hourly building loads based on the deep learning method. In recent years, the concept of meta-modeling, combining both the ideas of the physics-based method and data-driven method, has been introduced and applied to building simulation [23]. Zhang et al. [24] proposed a high-resolution meta-modeling framework for forecasting the hourly building electric load. Physics-based building energy models, namely meta-models, are simulated and calibrated to estimate the energy use of large-scale buildings. Advanced data mining techniques, including the machine learning algorithm, are trained with data generated from the physics-based models. The method performs well with high computational efficiency and prediction accuracy in two case studies of both residential and commercial building stock [24]. However, current studies focus more on forecasting the building energy consumption and pay less attention to the 
thermal demand, including cooling and heating loads, which are more instructive for the early design of building energy system. Ding et al. [25] collected and processed the energy data of 40 school buildings connected to the district heating system in Norway and proposed a systematic framework to forecast and identify the district heating load profiles of Nordic schools, combining the temperature moving average, correlation, and linear regression analysis. Validation results show that the defined load profiles represent the current energy demand well. The method does not require much time-consumed computation, so it can be transferred to other public buildings efficiently [25]. Considering that the recorded energy data may be not easy to obtain in some situations, instead, simulated load data from physics-based models are an essential part to be used for training the data-driven algorithm in our proposed meta-modeling workflow.

Figure 1 shows the structure of this paper. Cooling/heating load databases for different building types are firstly established as meta-models to train and test the deep learning model, while they consist of building features and annual hourly cooling and heating loads. The loads are obtained from batch simulation with the EnergyPlus program, which covers many thermal demand scenarios. On the basic of the load database, a one-dimensional convolutional neural network (1D-CNN) sequence-to-sequence (Seq2Seq) deep learning model is proposed to achieve forecasting. Finally, a district load forecasting framework is proposed, and this meta-modeling workflow is applied to an actual district case in Shanghai, China to forecast its all-year hourly cooling/heating loads.
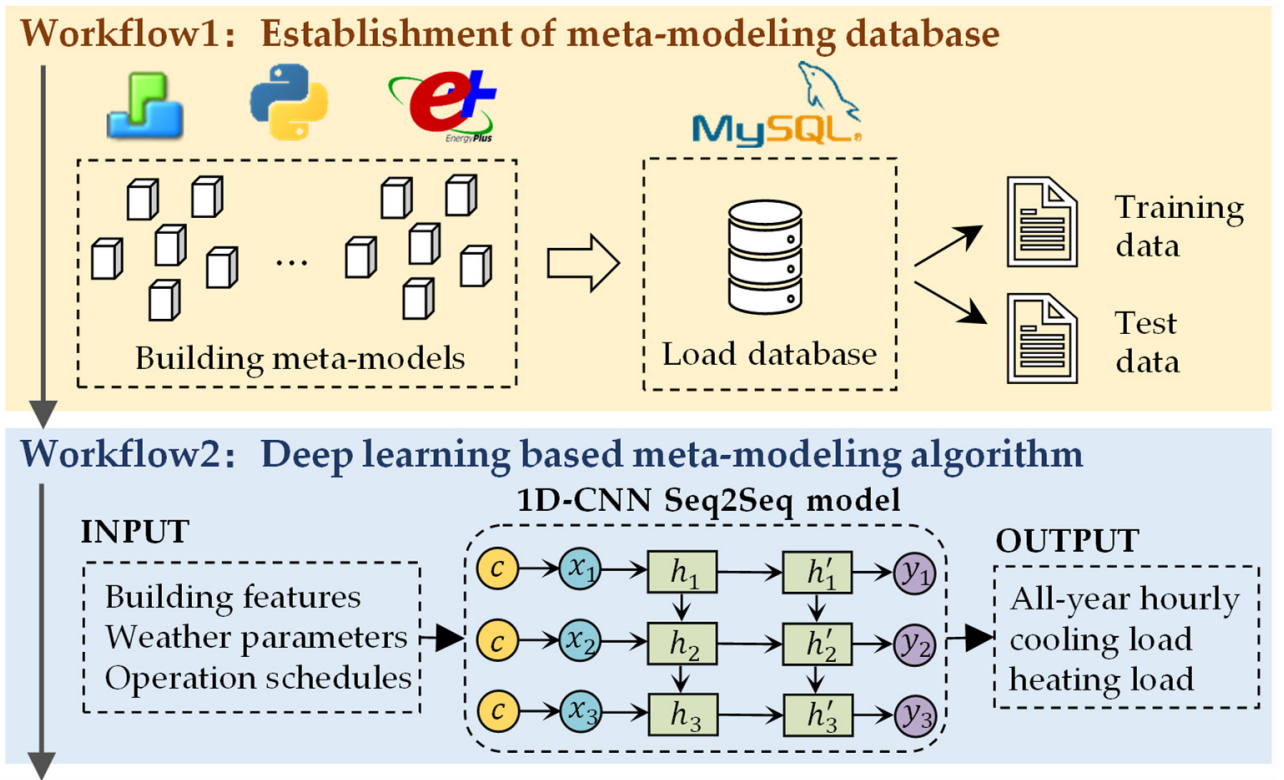

Workflow3: District load forecasting framework and case study

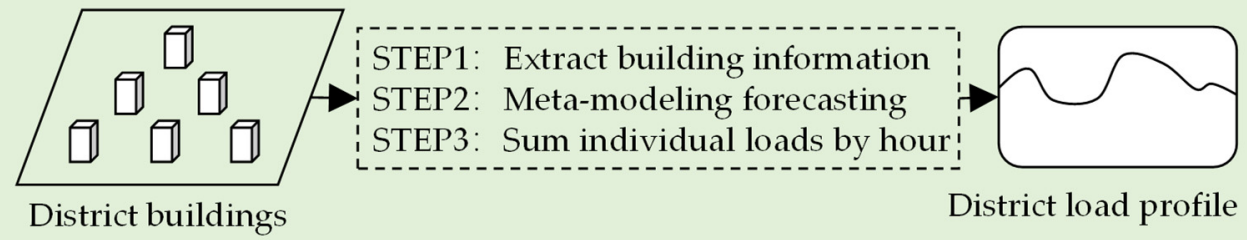

Figure 1. Structure of the meta-modeling workflow in this paper.

The main novelty of this paper is to propose a meta-modeling forecasting workflow for building cooling and heating loads with long period and high time resolution by hours throughout the year. The low-to-high dimensional mapping of load forecasting is transferred to be high-to-high dimensional, so that $1 \mathrm{D}-\mathrm{CNN}$ can be used as the metamodeling algorithm to lower the computing burden compared to other methods. Moreover, as thermal load forecasting for the district energy system is significant in the early design 
stage, a district load forecasting framework is innovatively proposed in this paper. By testing in a case study, it achieves fast and accurate load forecasting, with great reliability and convenience.

\section{Database}

\subsection{Building Feature Variables}

In order to comprehensively reflect the influence of building characteristics on the loads, it is necessary to extract the key building feature variables, which are non-negligible for building load forecasting in the design stage. Building loads include cooling, heating, and electrical loads, of which this paper focuses on building cooling and heating loads. Referring to an existing study of indispensable input variables for predicting building energy consumption [26], this paper selects seven building feature variables to establish the meta-model database. The seven variables are the building shape factor (BSF) representing the building geometry information, window-wall ratio (WWR) representing the envelope information, indoor design temperatures in summer $\left(T_{s}\right)$ and winter $\left(T_{w}\right)$, the occupancy density $\left(D_{0}\right)$, lighting power density $\left(D_{1}\right)$, and equipment power density $\left(D_{e}\right)$ representing the internal heat gain information. A comprehensive database should cover as many value levels of each independent variable as possible, but it is limited by the computing capacity and complexity. In this paper, the count of values for each variable is set as 4 , so as to reduce the computing workload and not to weaken the representativeness of the database. Table 1 shows the value range of each independent variable, which is obtained by expanding the standard value [27] for office, hotel, and commercial buildings.

Table 1. Value range of building feature variables.

\begin{tabular}{cccc}
\hline Classification & Variable & Unit & Values \\
\hline Building geometry & $\mathrm{BSF}$ & & $0.15,0.20,0.30,0.37$ \\
\hline Building envelope & $\mathrm{WWR}$ & $0.1,0.4,0.6,0.8$ \\
\hline \multirow{2}{*}{ Design temperature } & $\mathrm{T}_{\mathrm{s}}$ & ${ }^{\circ} \mathrm{C}$ & $22,24,26,28$ \\
\cline { 2 - 4 } & $\mathrm{T}_{\mathrm{w}}$ & ${ }^{\circ} \mathrm{C}$ & $16,18,20,21$ \\
\hline \multirow{2}{*}{ Internal heat gain } & $\mathrm{D}_{\mathrm{o}}$ & $\mathrm{m}^{2} / \mathrm{p}$ & $2,5,7,10$ (for office and hotel) \\
\cline { 2 - 4 } & & & $10,13,16,20$ (for commercial) \\
\cline { 2 - 4 } & $\mathrm{D}_{\mathrm{l}}$ & $\mathrm{W} / \mathrm{m}^{2}$ & $2,8,14,20$ \\
\hline & $\mathrm{D}_{\mathrm{e}}$ & $\mathrm{W} / \mathrm{m}^{2}$ & $2,8,14,20$ \\
\hline
\end{tabular}

\subsection{Meta-Model Simulation in Batches}

As shown in Table 2, cooling and heating loads are each described by six building feature variables, distinguished by the summer and winter indoor design temperature values. There are four possible values for each variable, which means the total number of building models is $8192\left(4^{6} \times 2\right)$. It is time-consuming to build these models one by one manually. As shown in Figure 2, this paper combines the manual and automatic approaches to generate the meta-models in batches and calculates their loads using EnergyPlus. The running file format in EnergyPlus is IDF, which essentially records the model information in text form. The automatic approach is to identify and modify the target variables in IDF files through automation code written by Python. However, it is difficult to achieve batch modification of the geometric variables related to the BSF and the WWR automatically, so manual modification is also needed. DesignBuilder is used to build 16 building geometry models shown in Figure 3 through modifying the BSF and the WWR manually. On the basis of geometry models, the remaining variables are modified by automatic approach to obtain the 8192 meta-models. 
Table 2. Variable sets of cooling and heating loads.

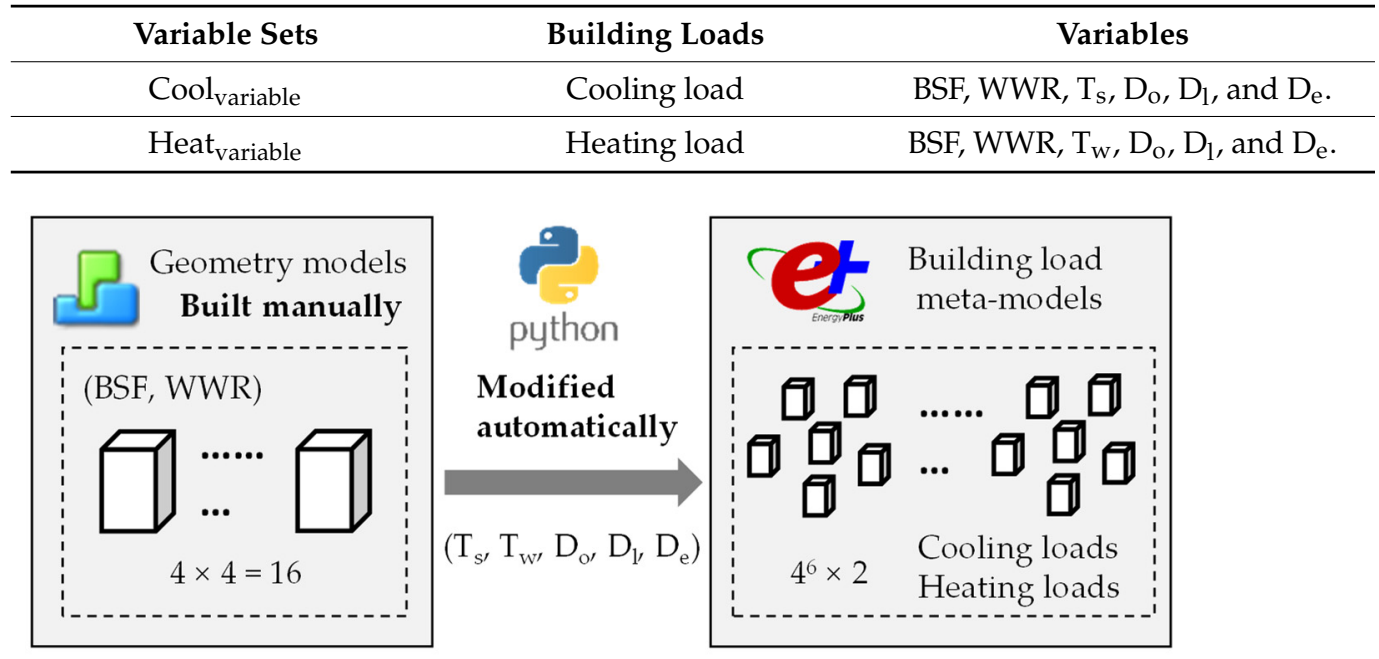

Figure 2. Approach to generate building meta-models.

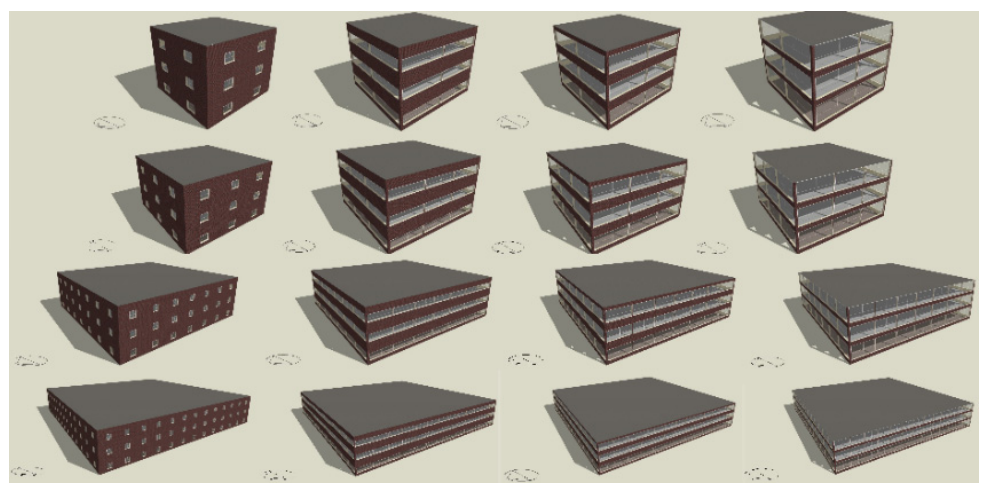

Figure 3. Sixteen building geometry models built in DesignBuilder.

After the batch generation of building meta-models, the next step is to calculate building hourly loads for each model in EnergyPlus. Besides building features, weather data and internal heat gain schedules are also needed for building hourly load simulation. Since the weather parameters are constantly changing, the typical meteorological year (TMY) is usually used as the input file of weather data in EnergyPlus. The weather files from Chinese standard weather data (CSWD) are selected, which provide hourly weather data in each region in China throughout the year. Internal heat gain schedules, including the occupancy schedule, lighting operation schedule, and equipment operation schedule, are set differently for three building types referring to the Chinese Design Standard for Energy Efficiency of Public Buildings (GB 50189-2015) [27].

\subsection{Database Establishment with MySQL}

According to the modeling approach, each meta-model contains seven building feature variables and $8760 \mathrm{~h}$ load results. This paper adopts MySQL to store and manage the database, as it performs well in storing relational data, such as building features and thermal loads. The most ideal storage mode of the database is to ensure each row corresponds to one meta-model, including seven-dimensional building information and 8760-dimensional load values. While MySQL can only support a maximum of 2048 fields in a row, it is necessary to propose a new data structure to store these variables-loads data.

As shown in Figure 4, this paper proposes split-table mode to convert each row in the ideal storage mode into a split data table, so as to overcome the row field limitation in MySQL. This method automatically creates a data table for each of the 8192 meta-models, 
which stores the cooling and heating loads corresponding to the time, with a dimension of $8760 \times 3$. To sum up, the database consists of one summary table and 8192 split tables, of which the summary table only includes seven variables' information of the 8192 models, with a dimension of $8192 \times 7$. The summary table is connected to each split table so that the load data can be extracted quickly and conveniently through corresponding building indexes in the summary table.

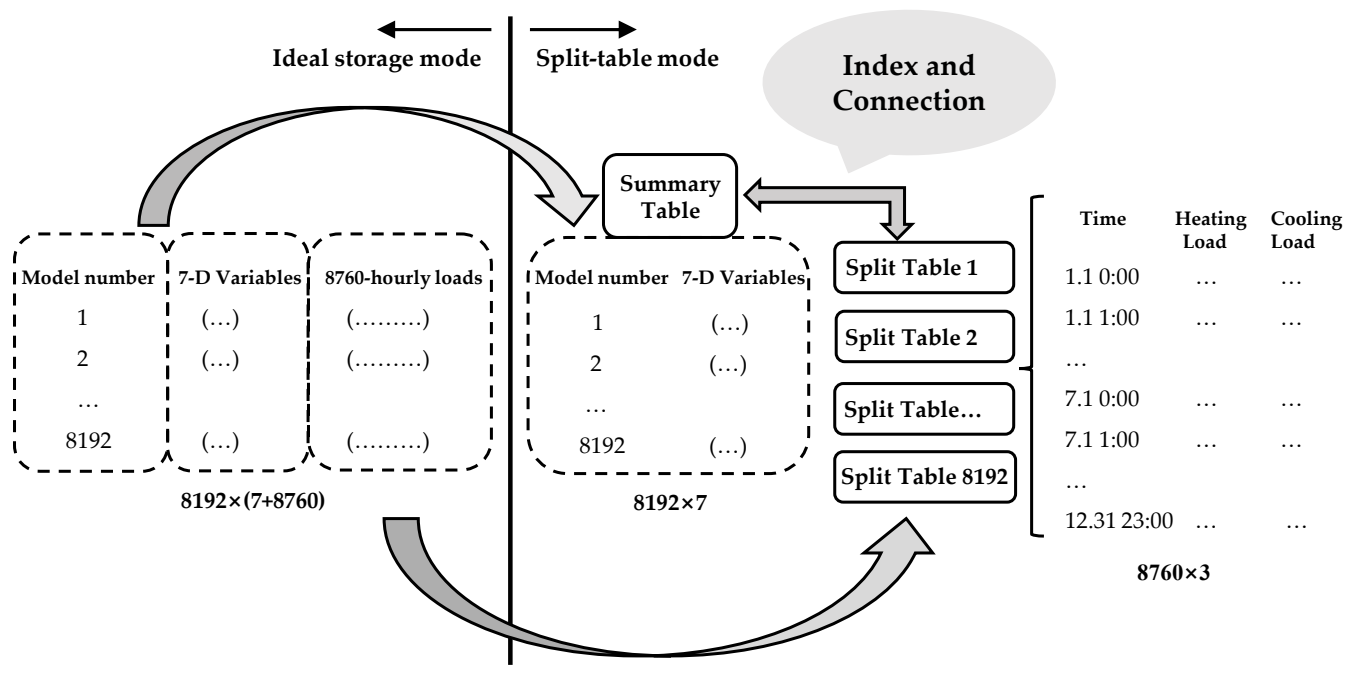

Figure 4. Split-table structure of database in MySQL.

\section{Methodology}

\subsection{Concept of Deep Learning}

As a kind of data-driven approximation of the detailed physics-based building models, a good meta-modeling workflow requires a well-performing data-mining algorithm. With the development of computer science, artificial intelligence (AI)-related research has begun to explode [28] (e.g., machine learning and deep learning), which not only appears in the field of computer science but also frequently in the engineering field [29,30]. Machine learning is to analyze data and learn from them to help forecast and make decisions [31]. Deep learning is not an independent learning method but a branch of machine learning [32].

In essence, the model of deep learning is a series of connected geometric change functions [33], which constantly act on the data. These functions are applied into each module, namely layers. The deep learning model consists of more layers than traditional shallow learning models in machine learning. Each layer contains representation and transformation rules for data, and these rules are parameterized by weights, which need to be learned in the training process. Due to its special network structure and excellent learning ability, deep learning performs better in analyzing image or sequence data than traditional machine learning methods $[34,35]$. Thus, in the proposed meta-modeling workflow of this paper, a sequence-to-sequence (Seq2Seq) deep learning model based on the one-dimensional convolutional neural network (1D-CNN) is chosen as the data-driven method to forecast the sequence data of long-term hourly loads.

\subsection{Task Design for Deep Learning}

Based on the variable-load database with meta-models, building loads forecasting can be regarded as a deep learning task with 7 building features as its input, and 8760 hours' cooling and heating loads as its output. For deep learning, it is difficult to deal with such a mapping relationship between a low-dimensional sequence and a highdimensional sequence. Therefore, it is necessary to transform the relationship between building features and hourly loads into a high-dimensional to high-dimensional mapping. 
On the one hand, the building has feature variables to describe the load response such as the 7 variables in this paper. On the other hand, the building loads also change with the weather parameters and operation schedules change in a year. Therefore, as time series data, there is a certain mapping relationship between the weather parameters and operation schedules, and the building loads at each hour, while this mapping relationship is determined by the building features. In a deep learning task, the building feature variables can be regarded as static variables, which describe the load response of the building under the action of weather parameters. The weather parameters of the building location and operation schedules of the building type can be regarded as dynamic variables, which act on the static variables of each building to obtain the all-year hourly loads. From this perspective, the learning task of the mapping relationship between 7-dimensional and 8760dimensional sequences is transformed into the learning task of the mapping relationship between 8760-dimensional and 8760-dimensional sequences, as shown in Figure 5.

7 to 8760

Low to high dimensional mapping

$\left(\mathrm{BSF}, \mathrm{WWR}, \mathrm{T}_{\mathrm{s}}, \mathrm{T}_{\mathrm{w}}, \mathrm{D}_{\mathrm{o}}, \mathrm{D}_{\mathrm{l}}, \mathrm{D}_{\mathrm{e}}\right)$

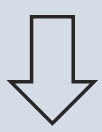

8760 hours' building loads
8760 to 8760

High to high dimensional mapping

\section{0 hours' dynamic variables}

Weather parameters

\& operation schedules

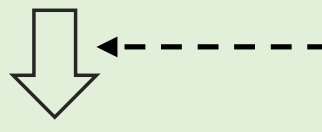

8760 hours' building loads

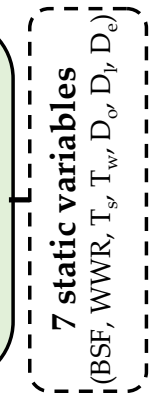

Figure 5. Mapping relationship transformation for deep learning.

This high-dimensional to high-dimensional mapping learning task is easier to realize using deep learning methods. In this paper, the dry-bulb temperature and solar radiation, which have a greater influence on loads, are selected as the dynamic variables related to weather parameters, and the occupancy schedule, lighting operation schedule, and equipment operation schedule are selected as the dynamic variables related to building types. In the same area, office buildings, hotels, and commercial buildings share the same weather dynamic variables, but their schedule dynamic variables are different, leading to the different overall dynamic variables. Under the same weather disturbance, the three different building types produce different load sequences.

\subsection{D-CNN Seq2Seq Model}

Each building has its own dynamic variables, static variables, and load profile. The relationship among them is what the deep learning model needs to learn, while the establishment of the learning model affects the forecasting performance greatly. It is essentially a sequence-to-sequence (Seq2Seq) task in the field of deep learning to learn the relationship between dynamic variables and static variables as the input and load profile as the output. The model to solve such tasks is called a Seq2Seq model, known as the encoder-decoder model, which is first proposed and applied in machine translation to convert a natural language (source domain) into another natural language (target domain) [36].

The most common structure for the Seq2Seq model is shown in Figure 6a, in which $x$ and $y$, respectively, represent the source domain sequence and target domain sequence of the model, $h$ and $h^{\prime}$ represent the hidden layer of the encoder and decoder, and $\mathrm{c}$ is the context vector. In the load forecasting task of this paper, the dynamic variables and load profile are the source and target domain sequence, respectively. The static variables can be regarded as the context vectors that describe the relationship between dynamic variables 
and loads. Therefore, a Seq2Seq model can realize the mapping learning of dynamic and static variables to loads.

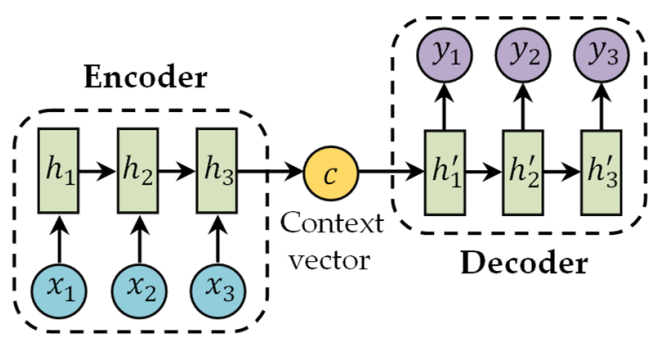

(a)

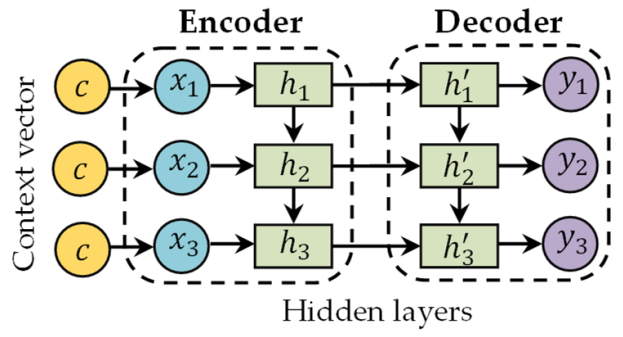

(b)

Figure 6. (a) Structure for Seq2Seq model; (b) Structure for 1D-CNN model.

The recurrent neural network (RNN) [37,38] and long short-term memory (LSTM) [39,40] are used as the encoder and decoder model in common Seq2Seq model. However, when they are trained, all the hidden layers of previous time steps must be kept, so the parallel computation inside the sequence cannot be realized. Only the sequential computation can be performed, which requires higher computing capacity. Recent studies [41,42] have adopted $\mathrm{CNN}$ to solve the Seq2Seq task. Bradbury et al. [43] propose recurrent pooling among a series of convolutional layers in 2016. Oord et al. [44] realize the character-to-character machine translation task using one-dimensional CNN (1D-CNN). Gehring et al. [45] add the attention mechanism to each convolution layer to realize the Seq2Seq translation task from German to English. The traditional CNN is a two-dimensional CNN (2D-CNN), which is mostly used to solve computer vision problems [46,47]. Its convolutional operation can extract features from the two-dimensional images and represent them in a modular way to make efficient use of input data. These characteristics make CNN perform well in the field of computer vision.

For temporal sequence problems such as load forecasting, CNN can also be used to solve them with the 1D-CNN, through regarding the time dimension as a spatial dimension (e.g., the height or the width of a two-dimensional picture). Owing to the convolution operation of $\mathrm{CNN}$, the calculation of each element in the sequence does not depend on the calculation of the previous time step. Therefore, for $1 \mathrm{D}-\mathrm{CNN}$, the parallel calculation of time sequence is feasible, and its computing burden is much lower than that of RNN and LSTM. Thus, this paper uses 1D-CNN to build a Seq2Seq model as the meta-modeling algorithm to solve the task of all-year hourly building load forecasting.

In the process of using 1D-CNN to build the Seq2Seq model, the static and dynamic variables are jointed as the inputs to the encoder. Then, the hidden layers are calculated as the input to the decoder to forecast the loads, as shown in Figure 6b. Different from Figure $6 a$, the static variables here are reflected at dynamic variables at each time, which helps the encoder and decoder better learn the quantitative effects of static variables in the mapping process.

Based on the above method, this paper uses Keras to establish the deep learning model. Keras is an open-source ANN library written in Python language and is widely used in academic fields due to its complete modularity and strong extensibility [48]. The detailed learning process is described in Figure 7, where the weather data, operation schedules, and building features are the input, and the all-year hourly building loads are the output of the model. The network framework of 1D-CNN Seq2Seq model established in Keras is summarized in Table 3, where the layers of Conv1d_1 and Conv1d_2 constitute the encoder, while Conv1d_3 and Conv1d_4 constitute the decoder. 


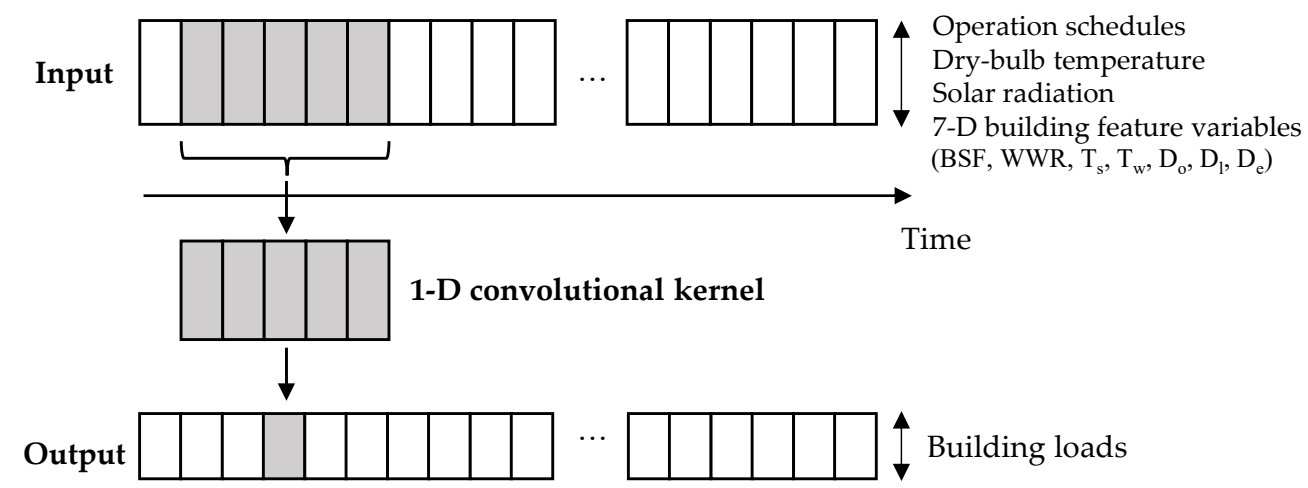

Figure 7. Learning process of the 1D-CNN Seq2Seq model.

Table 3. The network framework of 1D-CNN Seq2Seq model established in Keras.

\begin{tabular}{cccc}
\hline Structure & Network Layer & Layer Property & Dimension \\
\hline \multirow{2}{*}{ Input } & Input_var & InputLayer & $(168,12)$ \\
\cline { 2 - 4 } Encoder & Conv1d_1 & Conv1D & $(168,128)$ \\
\cline { 2 - 4 } & Conv1d_2 & Conv1D & $(168,128)$ \\
\cline { 2 - 4 } Decoder & Conv1d_3 & Conv1D & $(168,64)$ \\
\cline { 2 - 4 } & Conv1d_4 & Conv1D & $(168,32)$ \\
\hline \multirow{2}{*}{$\begin{array}{c}\text { Dropout } \\
\text { Output }\end{array}$} & Dropout_1 & Dropout & $(168,32)$ \\
\cline { 2 - 4 } & Load & Dense & $(168,1)$ \\
\hline
\end{tabular}

\subsection{Model Training and Evaluation}

After establishing the deep learning model, a sufficient training set and test set should be prepared to determine the parameters of each network layer and evaluate the model learning performance. For each building type, there are 8192 meta-models in the load database. According to the empirical law [49], a sampling proportion of $30 \%$ is sufficient to reflect the overall characteristics of database. Thus, 2500 models are randomly sampled to form the test set, whose loads, simulated using EnergyPlus, are regarded as the actual load values. The rest of the meta-models in the database form the training set for deep learning.

In detail, considering the network is too complex to be trained when the input and output are both 8760 dimensions, this paper adopts the idea of sequence splicing to establish the forecasting model with one week as the unit. The annual dynamic variables are disassembled into the dynamic variables with $52 \times 168 \mathrm{~h}$ (i.e., 52 weeks) to achieve forecasting, respectively, in which way the network input and output are both 168 dimensions. The forecasting process is performed 52 times in a year, and finally, the weekly load forecasting results are spliced together to obtain the all-year hourly loads. The whole process is shown in Figure 8, where the forecasting values from the 1D-CNN Seq2Seq model are compared with the actual values from simulation in the test set, to evaluate the deep learning performance. 


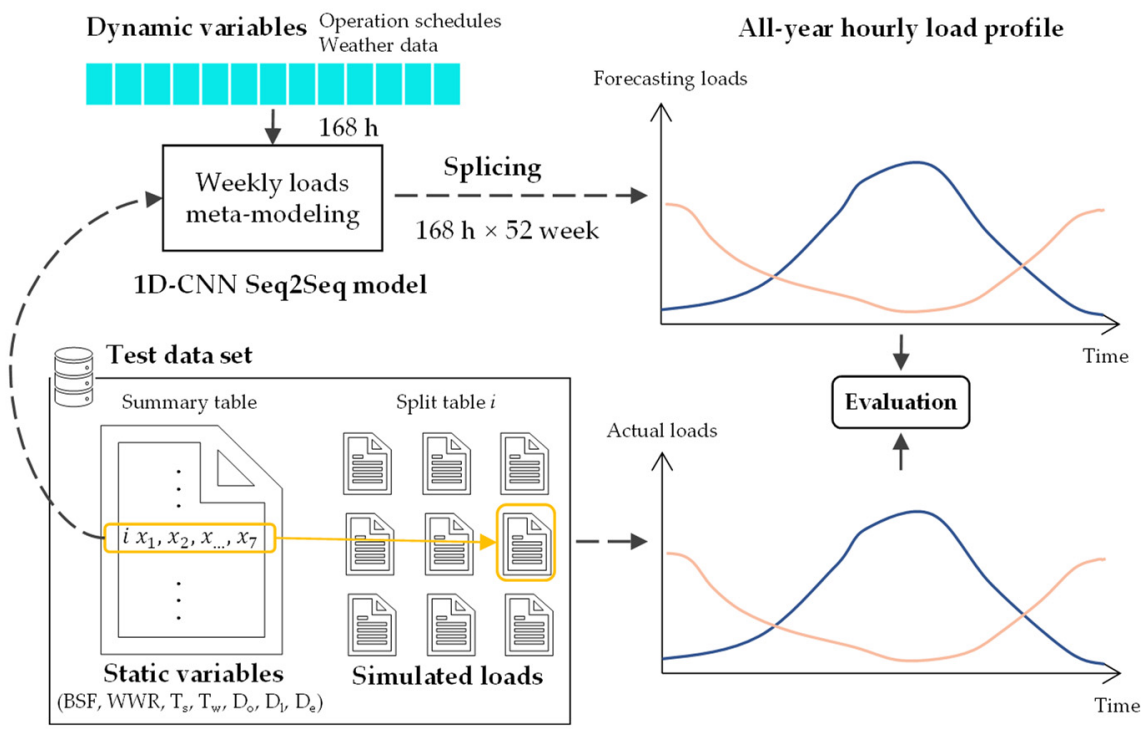

Figure 8. Forecasting process with the deep learning method.

For a conventional deep learning model, the loss can be a preliminary standard to evaluate the model training quality. Whether the model really learn the knowledge of the network may need to be evaluated by the metric on the test set. A commonly used evaluation metric is mean absolute percentage error (MAPE), calculated by Equation (1),

$$
\mathrm{MAPE}=\frac{1}{n} \sum_{t=1}^{n}\left|\frac{f_{t}-a_{t}}{a_{t}}\right| \times 100 \%
$$

where $f_{t}$ is the forecasting load at time $t, a_{t}$ is the actual load at time $t$, and $n$ represents the number of counted hours.

While calculating the MAPE value, the relative error of load forecasting at each hour is determined by Equation (2),

$$
\text { Relative Error }=\frac{f_{t}-a_{t}}{a_{t}} \times 100 \%
$$

where a negative relative error value means that the forecasting load is lower than the actual load, and a positive value means that the forecasting load is higher than the actual load at time $t$.

Moreover, considering that engineers pay more attention to the specific value than the profile of building load in the early design stage, this paper proposes an innovative metric, named the load effective hour rate (LEHR), to evaluate the similarity between the forecasting value and the actual value. The LEHR is calculated by Equation (3), which represents the proportion of hours with the forecasting error within $\pm 15 \%$ in the hours with non-zero load value throughout the year.

$$
\mathrm{LEHR}=\frac{N_{e}}{N} \times 100 \%
$$

where $N$ is the total number of hours in which the load is not zero in actual values, and $N_{e}$ is the number of effective hours, namely the number of hours in which the actual value is non-zero and relative error of forecasting value is in the interval of $[-15 \%, 15 \%]$.

\section{Results and Discussion}

Considering the different load profiles of different floors in a building, the building model is divided into three floors (the top floor, middle floor, and bottom floor). As shown in Figure 9, the top floor is exposed to solar radiation, while the bottom floor conducts heat 
with the ground. In the forecasting process of a multi-story building, the load values of the three floors will be forecasted separately and added together to obtain the total loads of the target building.

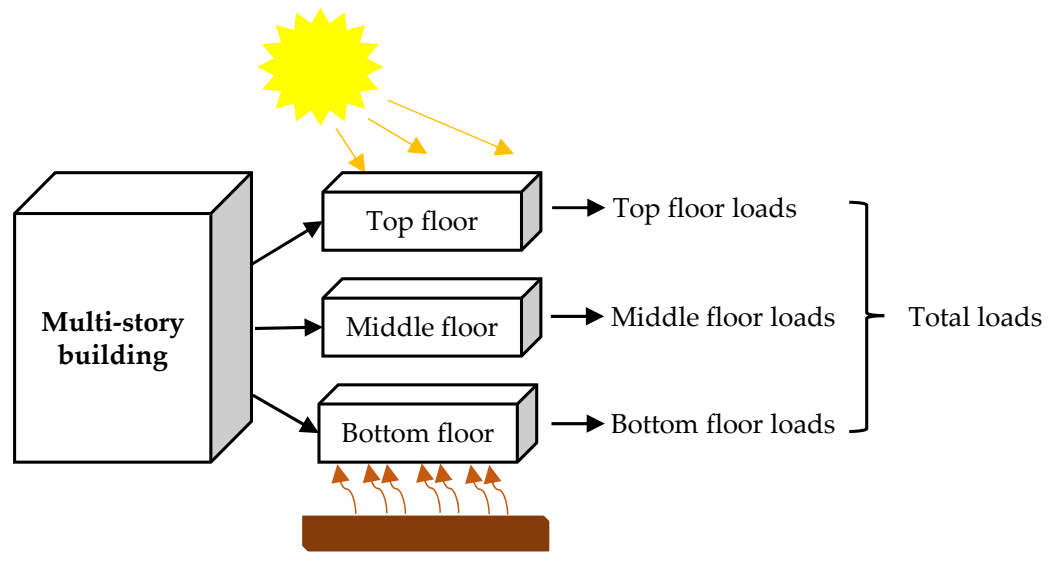

Figure 9. Floor division of multi-story buildings.

Tested with 2500 samples, cooling and heating LEHR values at the bottom, middle, and top floors of three different building types are evaluated. Taking office buildings as an example, Figure 10 shows the frequency distribution of cooling and heating LEHR values at different floors of 2500 samples, respectively. Most test samples fall in the interval of $[80 \%, 100 \%]$, showing a "peak phenomenon", which indicates that the overall forecasting performances are all good in different floors. The worst-performing test sample occurs in cooling load forecasting of the bottom floor, with the LEHR value less than $40 \%$.
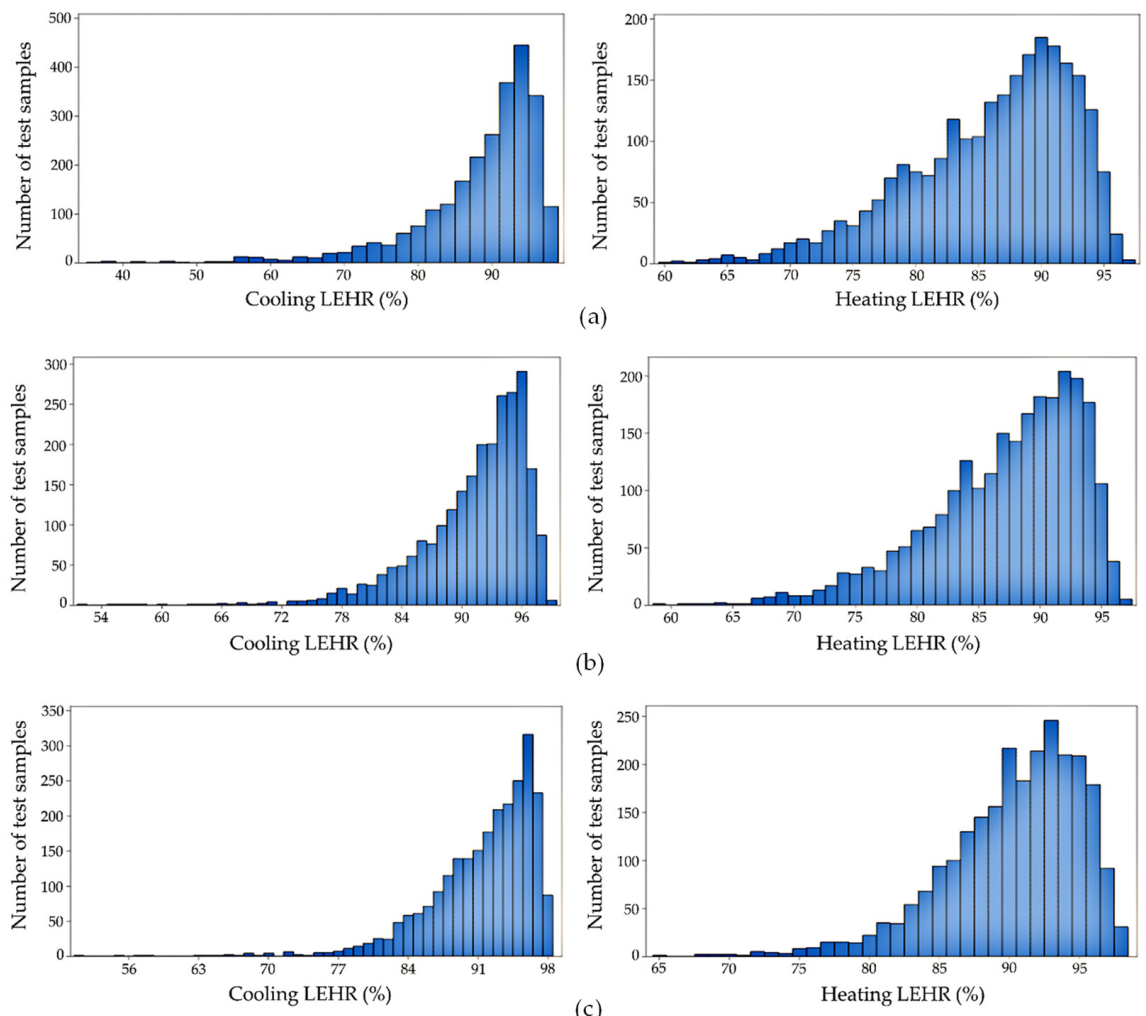

Figure 10. (a) LEHR distribution of bottom floors in office buildings; (b) LEHR distribution of middle floors in office buildings; (c) LEHR distribution of top floors in office buildings. 
Figure 11 shows the violin diagrams of cooling and heating LEHR values at different floors of 2500 samples for all three building types, respectively. For a particular building type, the distribution of LEHR values at the bottom, middle, and top floors is similar, which indicates that the mapping relationship between variables (static variable and dynamic variable) and load profile is essentially the same regardless of floors.

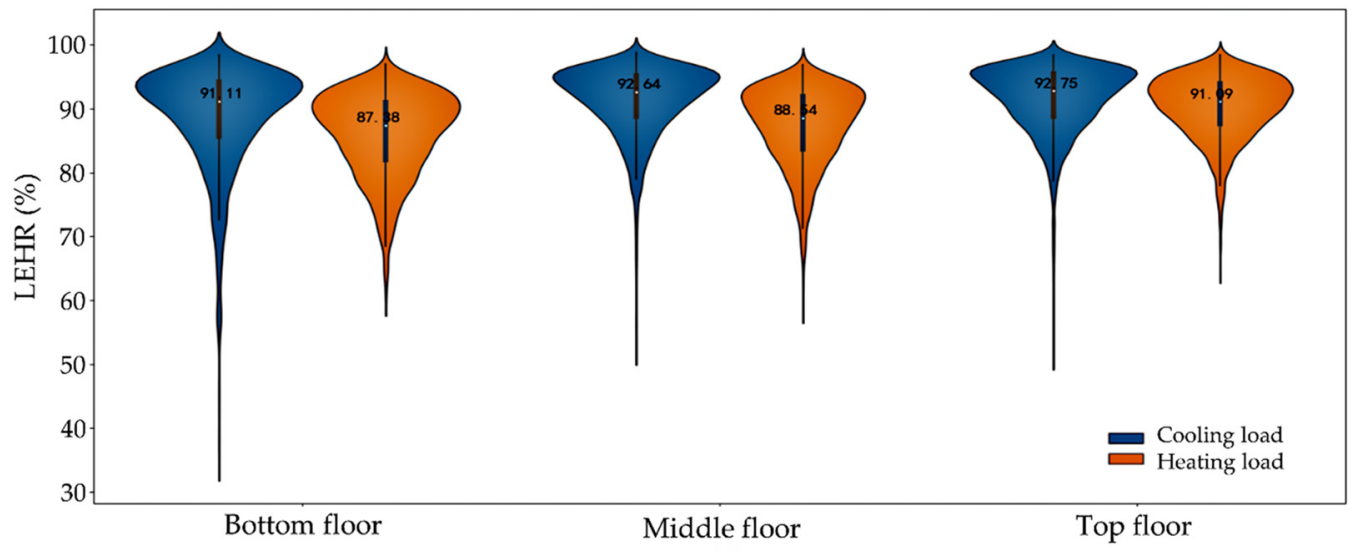

(a)

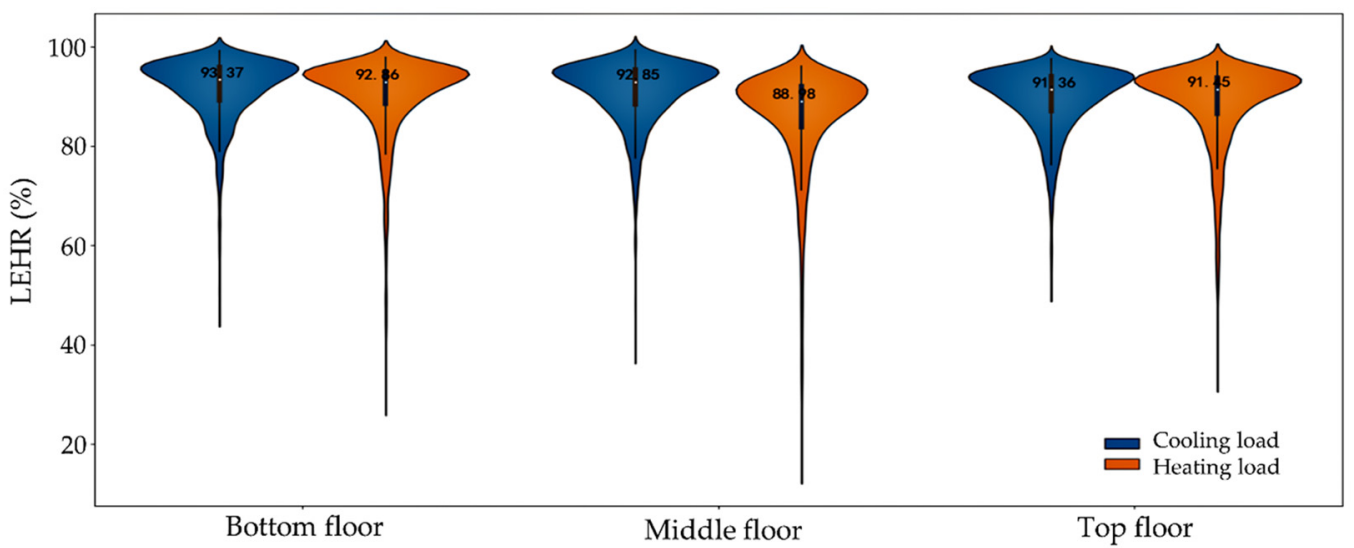

(b)

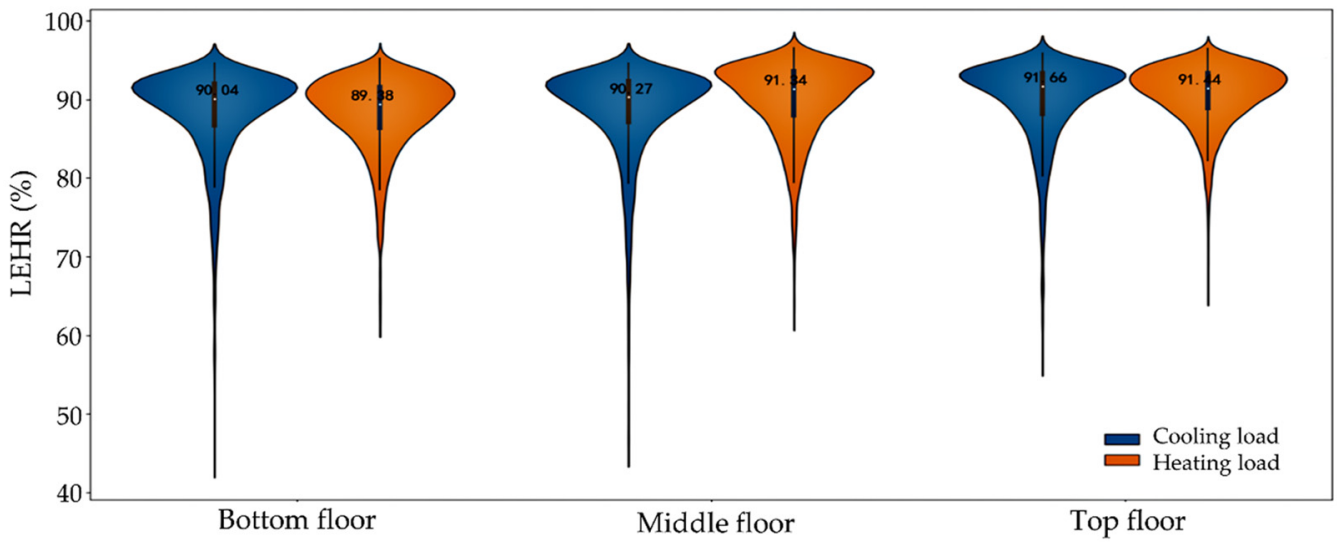

(c)

Figure 11. (a) LEHR distribution of office buildings; (b) LEHR distribution of commercial buildings; (c) LEHR distribution of hotels.

In particular, cooling LEHR distributions in office buildings and hotels show a longtail phenomenon, while heating LEHR distributions in commercial buildings show the long-tail phenomenon. According to the median LEHR value as shown in each diagram, 
the deep learning model performs a little better in forecasting the cooling load than heating. For three different building types, the model performs the best in hotels and the worst in office buildings. However, the distributions of LEHR values in three building types can still be regarded as similar, with only $0-5 \%$ differences on the median LEHR value, which illustrates the wide applicability of this method.

Table 4 shows the forecasting performance of three examples in 2500 test samples. The cooling LEHR (average value of three floors) is $91.1 \%$, and the heating LEHR (average value of three floors) is $89.4 \%$ for the office building. Cooling and heating LEHR for the hotel are $90.3 \%$ and $89.9 \%$, and $92.2 \%$ and $90.9 \%$ for the commercial building, respectively. In addition, MAPE of the cooling and heating forecasting loads are all less than $10 \%$ for the three examples. Referring to a previous mentioned study [25], where district heating load profiles are forecasted based on the collected energy information, an MAPE criterion less than $10 \%$ indicates a highly accurate forecasting quality, proving that the proposed 1D-CNN deep learning method can capture the building load profile well. Moreover, compared with the MAPE values evaluated in the referred paper [25], which are 20.2-29.6\% for forecasting district heating load profile and $6.6-8.8 \%$ for forecasting electricity load profile, the MAPE results in this paper show that the simulated load data from physics-based meta-models can be treated as an effective alternative when the recorded energy data are unavailable.

Table 4. Forecasting performance of three examples.

\begin{tabular}{ccccc}
\hline \multirow{2}{*}{ Building Type } & \multicolumn{2}{c}{ LEHR } & \multicolumn{2}{c}{ MAPE } \\
\cline { 2 - 5 } & Cooling Load & Heating Load & Cooling Load & Heating Load \\
\hline Office & $91.1 \%$ & $89.4 \%$ & $4.4 \%$ & $3.5 \%$ \\
\hline Hotel & $90.3 \%$ & $89.9 \%$ & $3.7 \%$ & $5.9 \%$ \\
\hline Commercial & $92.2 \%$ & $90.9 \%$ & $7.2 \%$ & $4.1 \%$ \\
\hline
\end{tabular}

\section{Case Study}

\subsection{District Building Loads Forecasting Framework}

The proposed meta-modeling method is developed from the load forecasting for an individual building. If it is applied to each building in a district, the district building load forecasting can be achieved by summing the all-year loads by hour according to each building area. The load values are summed hourly in the district load calculation, so the impact of different operation schedules on the load profile from different building types can be treated accurately with high resolution, which solves the over-estimation problem in the simple static superposition method. This paper proposes a district building load forecasting framework, as shown in Figure 12.

The district building load forecasting is split into three steps.

Step 1: Extract the information of the given district to obtain the building area, number of floors, and corresponding feature variables of each building in each type.

Step 2: Adopt the deep-learning-based meta-modeling method to carry out load forecasting for each building of each type throughout the year.

Step 3: Sum the loads hour by hour according to the area of each building in each type to obtain the overall all-year hourly building load profile of the district. 


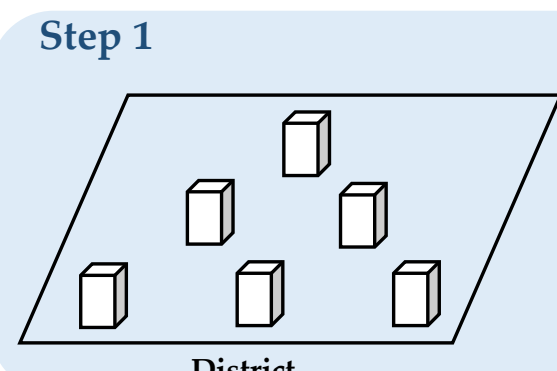

District

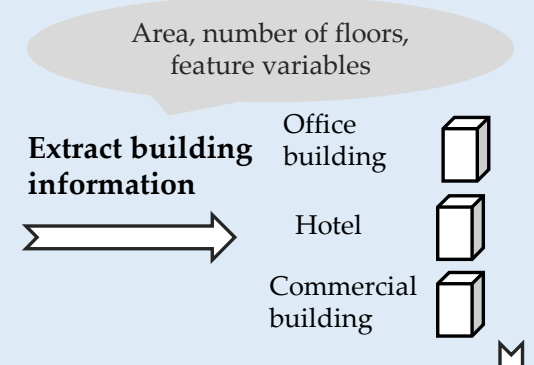

rea, number of floors,

feature variables

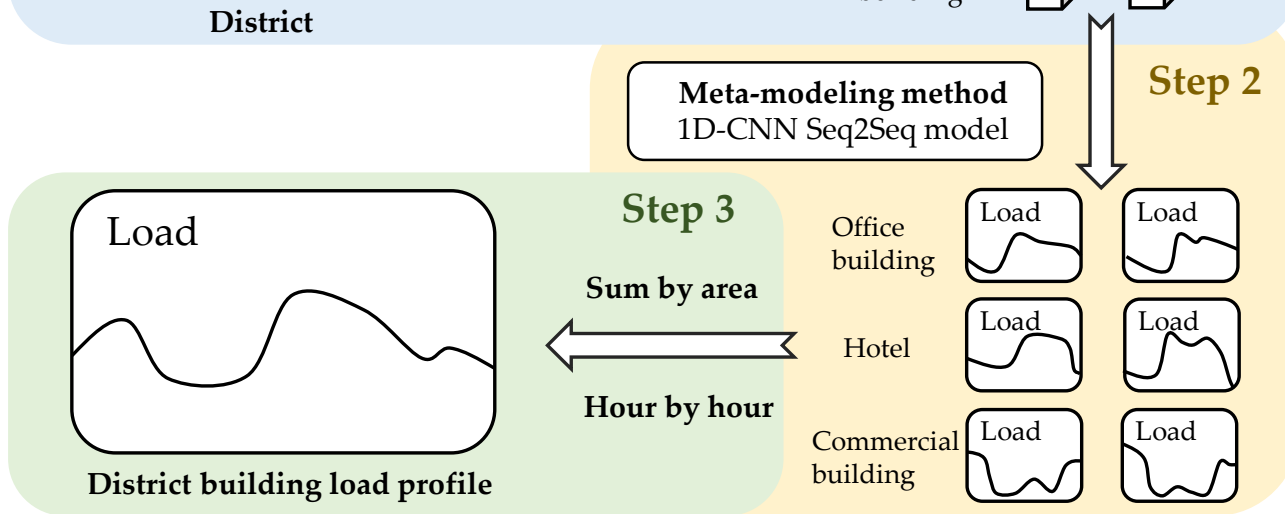

Figure 12. District building load forecasting framework.

\subsection{Load Forecasting Case for a Given District}

The meta-modeling workflow is applied to a case study of load forecasting in the early design stage for the west Hongqiao District, Shanghai. There are three buildings in this district shown in Figure 13, including a hotel, an office building, and a commercial building. Building \#1 is a hotel with height of $44.5 \mathrm{~m}$, ten floors, and a total building area of $19,890 \mathrm{~m}^{2}$. Building \#2 is an office building with height of $31.5 \mathrm{~m}$, seven floors, and a total building area of $17,301 \mathrm{~m}^{2}$. Building \#3 is a commercial building with height of $10.7 \mathrm{~m}$, two floors, and a total building area of $4430 \mathrm{~m}^{2}$. The input building feature variables are summarized in Table 5. Other features of buildings, such as operation schedules, specific types of walls and windows, and so on, are assumed as the same with the previously established 8192 meta-models, as they are unavailable in the district early design stage.

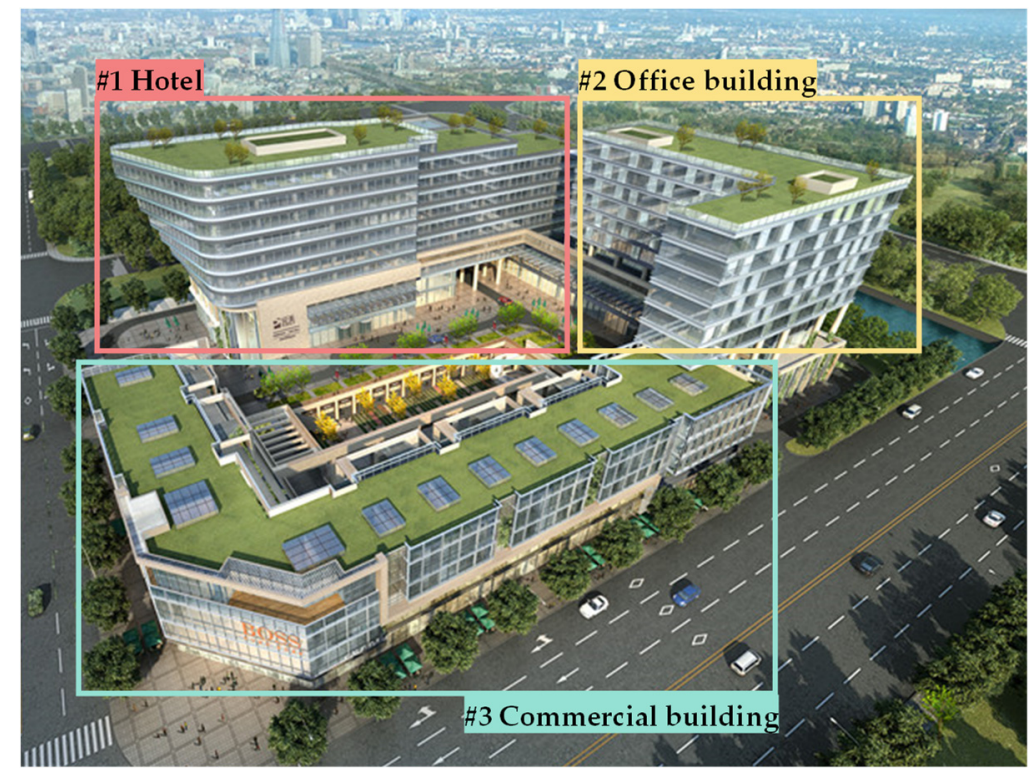

Figure 13. Studied case of the west Hongqiao District, Shanghai. 
Table 5. Building feature variables of the case.

\begin{tabular}{ccccccccc}
\hline \multirow{2}{*}{ No. } & \multirow{2}{*}{ Type } & BSF & WWR & $\mathbf{T}_{\mathbf{s}}$ & $\mathbf{T}_{\mathbf{w}}$ & $\mathbf{D}_{\mathbf{o}}$ & $\mathbf{D}_{\mathbf{l}}$ & $\mathbf{D}_{\mathbf{e}}$ \\
\cline { 3 - 9 } & & - & - & $\left({ }^{\circ} \mathbf{C}\right)$ & $\left({ }^{\circ} \mathbf{C}\right)$ & $\left(\mathbf{m}^{\mathbf{2}} \mathbf{p}\right)$ & $\left(\mathbf{W} / \mathbf{m}^{\mathbf{2}}\right)$ & $\mathbf{( \mathbf { W } / \mathbf { m } ^ { \mathbf { 2 } } )}$ \\
\hline$\# 1$ & hotel & 0.16 & 0.4 & 24 & 20 & 10 & 10 & 13 \\
\hline$\# 2$ & office & 0.18 & 0.5 & 26 & 20 & 6 & 12 & 20 \\
\hline$\# 3$ & commercial & 0.21 & 0.5 & 26 & 20 & 3 & 15 & 20 \\
\hline
\end{tabular}

Notations: $\mathrm{BSF}=$ building shape factor; $\mathrm{WWR}=$ window-wall ratio; $\mathrm{T}_{\mathrm{s}}=$ summer design temperature $\mathrm{T}_{\mathrm{W}}=$ winter design temperature; $\mathrm{D}_{\mathrm{o}}=$ occupancy density; $\mathrm{D}_{\mathrm{l}}=$ lighting power density; $\mathrm{D}_{\mathrm{e}}=$ equipment power density.

Figure 14a shows the actual load profile of this district, which is obtained from the simulation results in EnergyPlus. Figure 14b shows the forecasting hourly load result from the proposed meta-modeling workflow. Two of the load profiles are similar most of the time in a year. The distribution of hourly relative errors, calculated by Equation (2), is shown in Figure 15a, where all the cooling load errors and most of the heating load errors are within $\pm 15 \%$. The forecasting loads are slightly higher than actual loads at most hours with positive relative error values. Figure $15 \mathrm{~b}$ shows the scatter plot of actual and forecasting loads, and the forecasting values match the actual values well, with the coefficient of determination $\left(\mathrm{R}^{2}\right)$ of 0.9978 for heating load and 0.9975 for cooling load.

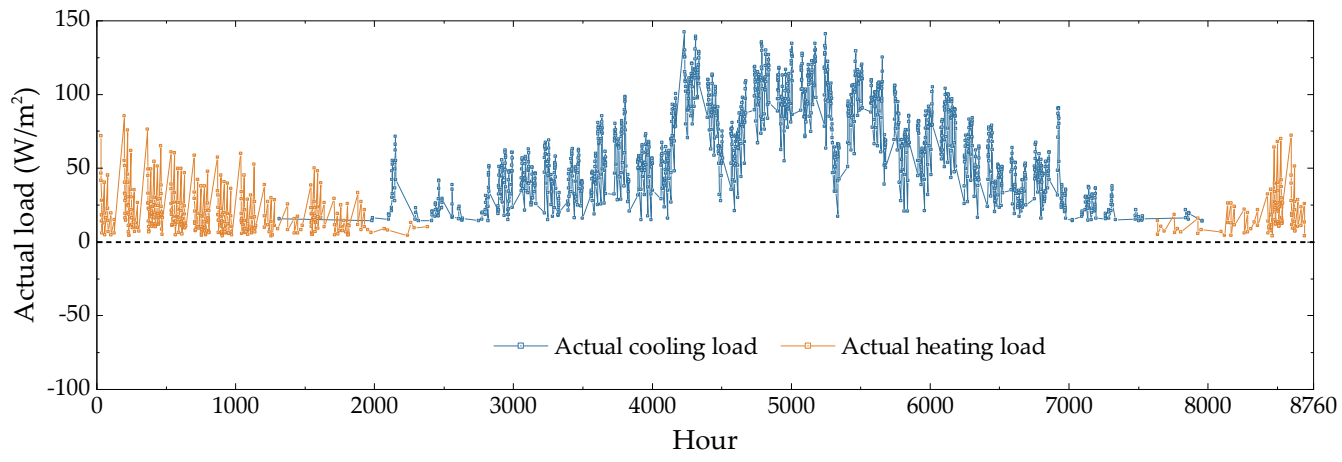

(a)

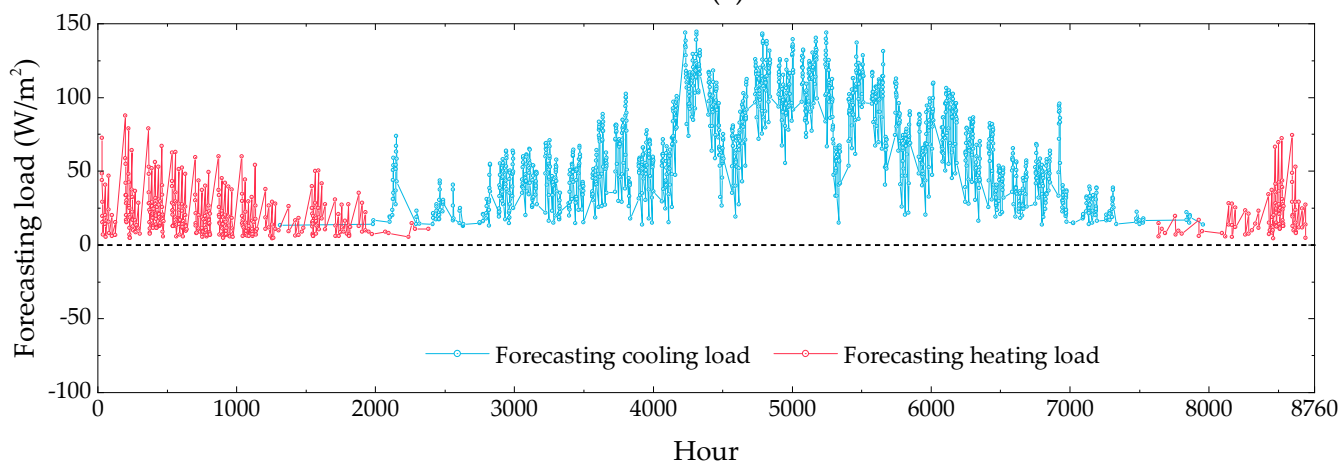

(b)

Figure 14. (a) Actual district load profile; (b) Forecasting district load profile. 


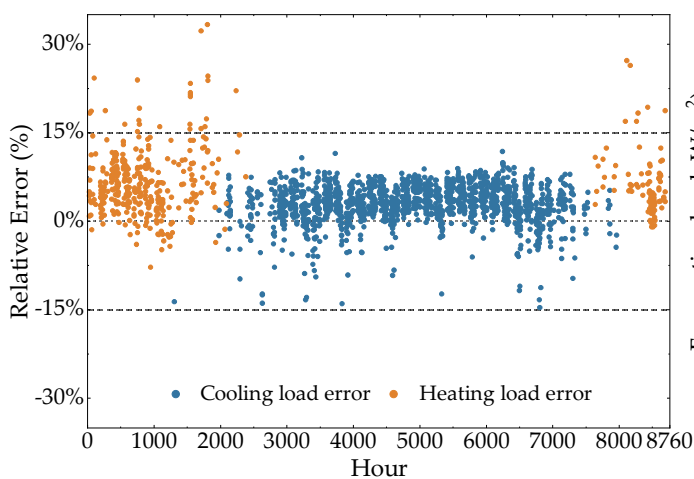

(a)

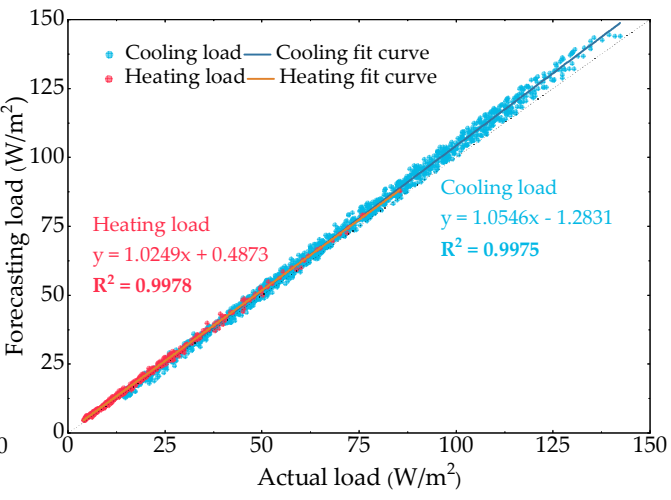

(b)

Figure 15. (a) Distribution of relative errors; (b) Scatter plot of forecasting and actual loads.

Table 6 summarizes the LEHR and MAPE values of the forecasting performance in this case study. As all the cooling load errors are within $\pm 15 \%$, the LEHR value of cooling load is $100.0 \%$. The LEHR value for all-year hourly load forecasting is $98.4 \%$. The MAPE value is $3.7 \%$ for the cooling load, $6.7 \%$ for the heating load, and $4.4 \%$ for the annual loads, respectively. In general, although the method performs better when forecasting cooling load than heating load in this case, it can be considered that the errors are all small enough to meet the precision requirement of district building load forecasting in the early design stage.

Table 6. Forecasting performance in the case study.

\begin{tabular}{ccc}
\hline Load Type & LEHR & MAPE \\
\hline Cooling load & $100.0 \%$ & $3.7 \%$ \\
\hline Heating load & $93.1 \%$ & $6.7 \%$ \\
\hline Annual loads & $98.4 \%$ & $4.4 \%$ \\
\hline Notations: LEHR = load effective hour rate & &
\end{tabular}

\section{Conclusions}

In this paper, a meta-modeling workflow is proposed to forecast the all-year hourly cooling and heating loads of building stock in the early design stage. As the basic of meta-modeling, load databases are firstly established for office, commercial, and hotel buildings. The database contains 8192 meta-models of each building type and stores their building feature variables and load values in MySQL with a split-table mode. Load values of all the meta-models are simulated in batches using EnergyPlus. As for the metamodeling algorithm, the low-dimensional to high-dimensional mapping learning task is firstly transferred to be high-dimensional to high-dimensional by introducing the static and dynamic variables. Thus, the forecasting task is more easily realized through deep learning methods. Considering a good performance in deep learning, the 1D-CNN Seq2Seq model is adopted, trained, and tested with sufficient simples from the database.

Evaluated by an innovative metric of LEHR and the MAPE between forecasting and actual loads, the meta-modeling workflow performs well at most times with an average LEHR of around $90 \%$ for all three building types, while the MAPE values are all less than $10 \%$. Through proposing the district building load forecasting framework, a district case in Shanghai is studied. Comparing the forecasting results with simulated actual loads, the LEHR value of all-year hourly loads is $98.4 \%$ and the MAPE is $4.4 \%$. It proves that the meta-modeling method meets the required precision of district building load forecasting in the early design stage and also reduces the computing burden to a great extent.

The proposed workflow achieves fast and accurate all-year hourly load forecasting in the building early design stage. It solves the deficiency of rough time resolution in current methods. Additionally, this meta-modeling workflow achieves load forecasting for 
buildings with simplified input variables, which expands the applicability of conventional physics-based methods. Moreover, the model is easily applied and time-saving for making decisions in the early design stage of the district building energy system.

Although the workflow performs well in forecasting building cooling and heating loads, it is limited by the selected input building features. Seven variables with significant impacts on the building thermal load are chosen as the input for meta-modeling in this paper. However, there are other factors such as the zoning of the buildings, types of walls and windows, and the particular operation schedules, which are not taken into consideration as they might be unavailable in the building early design stage. In addition, this study only focuses on three types of public buildings in a hot summer and cold winter area in China, and does not consider the varieties of climate region. As the proposed workflow has been verified with good performance, it can be further applied to other types of buildings under different climates.

Future works are mainly focused on two main directions. On the one hand, the effect of selecting input variables on the meta-modeling performance is worth studying to explore if there are any other essential building features as an input. On the other hand, the attention mechanism, which is commonly used in cutting-edge computer science [50], as well as other data-mining methods, can be combined with the 1D-CNN Seq2Seq model to help the workflow better capture building information and improve the forecasting accuracy.

Author Contributions: Y.Z.: Conceptualization, resources, data curation, project administration. Y.L.: methodology, writing-original draft preparation. Y.P.: methodology, writing-review and editing, supervision, project administration. X.Y.: methodology, writing-review and editing. Y.X.: resources, data curation. W.J.: conceptualization, methodology. All authors have read and agreed to the published version of the manuscript.

Funding: This research was funded by National Key R\&D Program of China, grant number No. 2018YFB0905101.

Institutional Review Board Statement: Not applicable.

Informed Consent Statement: Not applicable.

Data Availability Statement: Not applicable.

Conflicts of Interest: The authors declare no conflict of interest.

\section{References}

1. Huo, T.; Ma, Y.; Cai, W.; Liu, B.; Mu, L. Will the urbanization process influence the peak of carbon emissions in the building sector? A dynamic scenario simulation. Energy Build. 2021, 232, 110590. [CrossRef]

2. Hou, T.; Li, X.; Cai, W.; Zou, J.; Jia, F.; Wei, H. Exploring the impact of urbanization on urban building carbon emissions in China: Evidence from a provincial panel data model. Sustain. Cities Soc. 2020, 56, 102068.

3. IEA. Buildings: A Source of Enormous Untapped Efficiency Potential. Available online: https://www.iea.org/topics/buildings (accessed on 15 June 2021).

4. Sandberg, N.H.; Næss, J.S.; Brattebø, H.; Andresen, I.; Gustavsen, A. Large potentials for energy saving and greenhouse gas emission reductions from large-scale deployment of zero emission building technologies in a national building stock. Energy Policy 2021, 152, 112114. [CrossRef]

5. Rezaie, B.; Rosen, M.A. District heating and cooling: Review of technology and potential enhancements. Appl. Energy 2012, 93, 2-10. [CrossRef]

6. Jonshagen, K.; Genrup, M. Improved load control for a steam cycle combined heat and power plant. Energy 2010, 35, 1694-1700. [CrossRef]

7. Kavgic, M.; Mavrogianni, A.; Mumovic, D.; Summerfield, A.; Stevanovic, Z.; Djurovic-Petrovic, M. A review of bottom-up building stock models for energy consumption in the residential sector. Build. Environ. 2010, 45, 1683-1697. [CrossRef]

8. Ren, Z.; Paevere, P.; Grozev, G.; Egan, S.; Anticev, J. Assessment of end-use electricity consumption and peak demand by Townsville's housing stock. Energy Policy 2013, 61, 888-893. [CrossRef]

9. Ren, Z.; Paevere, P.; Mcnamara, C. A local-community-level, physically-based model of end-use energy consumption by Australian housing stock. Energy Policy 2012, 49, 586-596. [CrossRef]

10. Johnston, D. A Physically Based Energy and Carbon Dioxide Emission Model of the UK Housing Stock; Leeds Metropolitan University: Leeds, UK, 2003; pp. 11-18. 
11. Heiple, S.; Sailor, D.J. Using building energy simulation and geospatial modeling techniques to determine high resolution building sector energy consumption profiles. Energy Build. 2008, 40, 1426-1436. [CrossRef]

12. Di Leo, S.; Caramuta, P.; Curci, P.; Cosmi, C. Regression analysis for energy demand projection: An application to TIMES-Basilicata and TIMES-Italy energy models. Energy 2020, 196, 117058. [CrossRef]

13. Ramanathan, R.; Engle, R.; Granger, C.W.J.; Vahid-Araghi, F.; Brace, C. Short-run forecasts of electricity loads and peaks. Int. J. Forecast. 1997, 13, 161-174. [CrossRef]

14. Dotzauer, E. Simple model for prediction of loads in district-heating systems. Appl. Energy 2002, 73, 277-284. [CrossRef]

15. Vaghefi, A.; Jafari, M.A.; Bisse, E.; Lu, Y.; Brouwer, J. Modeling and forecasting of cooling and electricity load demand. Appl. Energy 2014, 136, 186-196. [CrossRef]

16. Barakat, E.H.; Qayyum, M.A. Short-term peak demand forecasting in fast developing utility with inherit dynamic load characteristics. I. Application of classical time-series methods. II. Improved modelling of system dynamic load characteristics. Power Syst. IEEE Trans. 1990, 5, 813-824. [CrossRef]

17. Ogunsola, O.T.; Song, L.; Wang, G. Development and validation of a time-series model for real-time thermal load estimation. Energy Build. 2014, 76, 440-449. [CrossRef]

18. Dong, B.; Cao, C.; Lee, S.E. Applying support vector machines to predict building energy consumption in tropical region. Energy Build. 2005, 37, 545-553. [CrossRef]

19. Al-Shammari, E.T.; Keivani, A.; Shamshirband, S.; Mostafaeipour, A.; Yee, P.L.; Petković, D.; Ch, S. Prediction of heat load in district heating systems by Support Vector Machine with Firefly searching algorithm. Energy 2016, 95, 266-273. [CrossRef]

20. Yu, W.; Li, B.; Lei, Y.; Liu, M. Analysis of a Residential Building Energy Consumption Demand Model. Energies 2011, 4, 475-487. [CrossRef]

21. Vázquez-Canteli, J.R.; Dilsiz, A.D.; Brown, J.; Nagy, Z. Deep neural networks as surrogate models for urban energy simulations. J. Phys. Conf. Ser. 2019, 1343, 012002. [CrossRef]

22. Hou, Z.; Lian, Z.; Yao, Y.; Yuan, X. Cooling-load prediction by the combination of rough set theory and an artificial neural-network based on data-fusion technique. Appl. Energy 2006, 83, 1033-1046. [CrossRef]

23. Westermann, P.; Evins, R. Surrogate modelling for sustainable building design-A review. Energy Build. 2019, 198, 170-186. [CrossRef]

24. Zhang, L.; Plathottam, S.; Reyna, J.; Merket, N.; Sayers, K.; Yang, X.; Reynolds, M.; Parker, A.; Wilson, E.; Fontanini, A.; et al High-resolution hourly surrogate modeling framework for physics-based large-scale building stock modeling. Sustain. Cities Soc. 2021, 75, 103292. [CrossRef]

25. Ding, Y.; Brattebø, H.; Nord, N. A systematic approach for data analysis and prediction methods for annual energy profiles: An example for school buildings in Norway. Energy Build. 2021, 247, 111160. [CrossRef]

26. Zhu, M.; Pan, Y.; Huang, Z. The Construction of Minimum Variables Set for Energy Prediction Models of Office Buildings. In Proceedings of the 16th IBPSA Conference, Rome, Italy, 2-4 September 2019; pp. 4157-4164. [CrossRef]

27. GB 50189-2015; Design Standard for Energy Efficiency of Public Buildings. Ministry of Housing and Urban-Rural Development of the People's Republic of China (MOHURD): Beijing, China, 2015. Available online: https://img.antpedia.com/standard/files/ pdfs_ora/CN-GB/b01/GB\%2050189-2015_2496.pdf(accessed on 1 January 2022).

28. Van der Maas, H.L.J.; Snoek, L.; Stevenson, C.E. How much intelligence is there in artificial intelligence? A 2020 update. Intelligence 2021, 87, 101548. [CrossRef]

29. Zeba, G.; Dabić, M.; Čičak, M.; Daim, T.; Yalcin, H. Technology mining: Artificial intelligence in manufacturing. Technol. Forecast. Soc. Chang. 2021, 171, 120971. [CrossRef]

30. Chen, C.; Hu, Y.; Karuppiah, M.; Kumar, P.M. Artificial intelligence on economic evaluation of energy efficiency and renewable energy technologies. Sustain. Energy Technol. Assess. 2021, 47, 121358. [CrossRef]

31. Sircar, A.; Yadav, K.; Rayavarapu, K.; Bist, N.; Oza, H. Application of machine learning and artificial intelligence in oil and gas industry. Pet. Res. 2021, 6, 379-391. [CrossRef]

32. Khallaf, R.; Khallaf, M. Classification and analysis of deep learning applications in construction: A systematic literature review. Autom. Constr. 2021, 129, 103760. [CrossRef]

33. Dong, S.; Wang, P.; Abbas, K. A survey on deep learning and its applications. Comput. Sci. Rev. 2021, 40, 100379. [CrossRef]

34. Liu, Z.; Jin, L.; Chen, J.; Fang, Q.; Ablameyko, S.; Yin, Z.; Xu, Y. A survey on applications of deep learning in microscopy image analysis. Comput. Biol. Med. 2021, 134, 104523. [CrossRef]

35. Bai, X.; Wang, X.; Liu, X.; Liu, Q.; Song, J.; Sebe, N.; Kim, B. Explainable deep learning for efficient and robust pattern recognition: A survey of recent developments. Pattern Recognit. 2021, 120, 108102. [CrossRef]

36. Ma, Z.; Du, B.; Shen, J.; Yang, R.; Wan, J. An Encoding Mechanism for Seq2Seq based Multi-Turn Sentimental Dialogue Generation Model. Procedia Comput. Sci. 2020, 174, 412-418. [CrossRef]

37. Li, M.; Miao, Z.; Xu, W. A CRNN-based attention-seq2seq model with fusion feature for automatic Labanotation generation. Neurocomputing 2021, 454, 430-440. [CrossRef]

38. Yu, W.; Kim, I.Y.; Mechefske, C. Analysis of different RNN autoencoder variants for time series classification and machine prognostics. Mech. Syst. Signal Processing 2021, 149, 107322. [CrossRef]

39. Yin, H.; Zhang, X.; Wang, F.; Zhang, Y.; Xia, R.; Jin, J. Rainfall-runoff modeling using LSTM-based multi-state-vector sequence-tosequence model. J. Hydrol. 2021, 598, 126378. [CrossRef] 
40. Fang, Z.; Crimier, N.; Scanu, L.; Midelet, A.; Alyafi, A.; Delinchant, B. Multi-zone indoor temperature prediction with LSTM-based sequence to sequence model. Energy Build. 2021, 245, 111053. [CrossRef]

41. Gao, Y.; Ruan, Y.; Fang, C.; Yin, S. Deep learning and transfer learning models of energy consumption forecasting for a building with poor information data. Energy Build. 2020, 223, 110156. [CrossRef]

42. Zhang, G.; Bai, X.; Wang, Y. Short-time multi-energy load forecasting method based on CNN-Seq2Seq model with attention mechanism. Mach. Learn. Appl. 2021, 5, 100064. [CrossRef]

43. Bradbury, J.; Merity, S.; Xiong, C.; Socher, R. Quasi-Recurrent Neural Networks. arXiv 2016, arXiv:1611.01576.

44. Oord, A.; Kalchbrenner, N.; Vinyals, O.; Espeholt, L.; Graves, A.; Kavukcuoglu, K. Conditional image generation with pixelcnn decoders. arXiv 2016, arXiv:1606.05328.

45. Gehring, J.; Auli, M.; Grangier, D.; Yarats, D.; Dauphin, Y.N. Convolutional Sequence to Sequence Learning. In Proceedings of the 34th International Conference on Machine Learning, Sydney, Australia, 6-11 August 2017. Available online: http: // proceedings.mlr.press /v70/gehring17a/gehring17a.pdf (accessed on 1 January 2022).

46. Zhang, K.; Wang, W.; Lv, Z.; Fan, Y.; Song, Y. Computer vision detection of foreign objects in coal processing using attention CNN. Eng. Appl. Artif. Intell. 2021, 102, 104242. [CrossRef]

47. Yang, R.; Singh, S.K.; Tavakkoli, M.; Amiri, N.; Yang, Y.; Karami, M.A.; Rai, R. CNN-LSTM deep learning architecture for computer vision-based modal frequency detection. Mech. Syst. Signal Processing 2020, 144, 106885. [CrossRef]

48. Conlin, R.; Erickson, K.; Abbate, J.; Kolemen, E. Keras2c: A library for converting Keras neural networks to real-time compatible C. Eng. Appl. Artif. Intell. 2021, 100, 104182. [CrossRef]

49. Neuman, W.L. Social Research Methods: Qualitative and Quantitative Approaches, 7th ed.; Pearson: Essex, UK, 2014. Available online: https:/ / www.scirp.org/(S(lz5mqp453edsnp55rrgjct55))/ reference/referencespapers.aspx?referenceid=2744295 (accessed on 1 January 2022).

50. Niu, Z.; Zhong, G.; Yu, H. A review on the attention mechanism of deep learning. Neurocomputing 2021, 452, 48-62. [CrossRef] 